\title{
Architecture Engineering of One Body \\ Nanostructured Catalyst: Layer-By-Layer Adornment of Multiple Nanocatalysts On Silica Nanorod Arrays For Hydrogenation of Nitroarenes
}

Kootak Hong

Seoul National University

Jun Min Suh

Seoul National University

Tae Hyung Lee

Seoul National University

Seeram Ramakrishna

National University of Singapore

Rajender S. Varma

Palacký University

Ho Won Jang

Seoul National University

Mohammadreza Shokouhimehr ( $\nabla$ mrsh2@snu.ac.kr)

Seoul National University

\section{Research Article}

Keywords: Catalysis, Nanostrucutre, Hydrogenation, Reduction, Coupling reaction

Posted Date: July 7th, 2021

DOI: https://doi.org/10.21203/rs.3.rs-658873/v1

License: (c) (i) This work is licensed under a Creative Commons Attribution 4.0 International License. Read Full License 


\section{Abstract}

Direct consideration for both, the catalytically active species and the host materials provides highly efficient strategies for the architecture design of nanostructured catalysts. The conventional wet chemical methods have limitations in achieving such unique layer-by-layer design possessing one body framework with many catalyst parts. Herein, an innovative physical method is presented that allows the wellregulated architecture design for an array of functional nanocatalysts as exemplified by layer-by-layer adornment of Pd nanoparticles (NPs) on the highly arrayed silica nanorods. This spatially confined catalyst exhibits excellent efficiency for the hydrogenation of nitroarenes and widely deployed Suzuki cross-coupling reactions; their facile separation from the reaction mixtures is easily accomplished due to the monolithic structure. The generality of this method for the introduction of other metal source has also been demonstrated with Au NPs. This pioneering effort highlights the feasibility of physically controlled architecture design of nanostructured catalysts which may stimulate further studies in the general domain of the heterogeneous catalytic transformations.

\section{Introduction}

Catalysts are sine qua non for most of the chemical processes to produce fine chemicals, pharmaceuticals, petrochemicals, among plethora of other fine chemicals. ${ }^{1-7}$ From the industrial point of view, heterogeneous catalysts are often preferred to their homogeneous counterparts because of low cost, easy separation from reaction mixture, stability, and reusability. ${ }^{8-10}$ In addition, the use of heterogeneous catalysts in continuous flow processes have several advantageous features such as easy mass production, automation, higher yields, and quality of products. ${ }^{11-14}$

Despite these remarkable advantages, one of the critical issues in heterogeneous catalysts is their low catalytic activity and selectivity. ${ }^{15-17}$ In particular, very low catalytic activity and the rapid decay in efficiency are inevitable when they are stabilized in the non-porous solid supports. Therefore, chemical and material engineers have made several strides to design and synthesize heterogeneous catalysts with enhanced catalytic activity and selectivity. As supported heterogeneous catalysts are composed of two

important segments: (i) catalytically active metal nanoparticles (NPs), and (ii) support materials. ${ }^{18-20}$, the developed methodologies to enhance the catalytic activity of metal NPs entails tailoring their morphologies, facets, and atomic arrangements. ${ }^{21-24}$ Support materials have also been extensively developed to uncover facile recyclability, good physiochemical stability, high surface area for the accessibility of metal nanocatalysts to various substrates and reactants. ${ }^{25-28}$ In addition, various approaches have been explored to functionalize the engineered heterogeneous catalysts thus enabling them to accommodate high loading capacity of catalytically active nanocatalysts and ameliorating their performance. ${ }^{29-34}$

For the next generation of heterogeneous catalysts endowed with specific functions and high catalytic activity, it is indispensable to design the architecture of engineered catalytically active NPs to be 
deposited in the accessible positions. Undeniably, the catalytic systems often developed by wet chemistry, the widely used method to prepare heterogeneous catalysts, cannot immobilize multiple

catalytically active NPs on supports in a controllable manner at the designed nanoscale. ${ }^{35-38}$ Even if endeavored, it requires very complicated and time-consuming multi-step process and the deployment of toxic chemical reagents. Therefore, new methods of designing and fabricating the heterogeneous catalysts comprising unique frameworks with various components should be planned.

Physically controlled decoration of catalytically active species on certain well-known and defined supports provides a great potential to fulfill this objective. Compared to the conventional wet-chemical methods, this strategy presents advantages of well-tolerated loading amounts and decoration locations on supports, which is unattainable by existing synthetic techniques. Consequently, we established in our approach the highly tolerable design of benign hosts and catalytically active species.

A novel template-free and designable bottom-up grown Pd NPs deposited on vertically aligned $\mathrm{SiO}_{2}$ nanorod arrays ( $\mathrm{v}-\mathrm{SiO}_{2} \mathrm{NRs@Pd)} \mathrm{nanostructured} \mathrm{catalyst} \mathrm{(prepared} \mathrm{by} \mathrm{glancing} \mathrm{angle} \mathrm{deposition} \mathrm{(GLAD)}$ method) is reported for the reduction of nitroarenes. Due to shadowing effect during the GLAD process, $\mathrm{SiO}_{2} \mathrm{NRs}$ can be deposited on silicon wafers. As $\mathrm{SiO}_{2} \mathrm{NRs}$ have top open structure, accessible Pd NPs can be precisely decorated on the surface of $\mathrm{SiO}_{2} \mathrm{NRs}$ in a layer-by-layer architecture benefitting from a multiple-step GLAD method. Furthermore, our strategy enables the combinations of various metal NPs and support materials, and nano-scale controlled affixation of catalyst NPs robustly on nanorod arrays.

\section{Materials And Methods}

Oxide grains $\left(\mathrm{SiO}_{2}\right.$ and $\left.\mathrm{SnO}_{2}\right)$ and metal pellets ( $\mathrm{Pd}$ and $\mathrm{Au}$ ) with $99.99 \%$ purity, used for film deposition, were purchased from Kojundo Chemistry. The other chemicals used in this study were purchased from the Daejung and Samchun Chemical Companies.

Fabrication of the v-SiO 2 NRs@Pd nanostructured catalyst: A $p$-type Si wafers were cleaned in acetone, isopropanol, and deionized water under ultrasonication followed by drying in a nitrogen atmosphere. To make $\mathrm{SiO}_{2} \mathrm{NRs}$, the evaporation was performed at a glancing angle with a rotation speed of $40 \mathrm{rpm}$; the wafer was located $50 \mathrm{~cm}$ away from the crucible, tilted at $80^{\circ}$. The base pressure was maintained at $1.0 \times$ $10^{-6}$ Torr and growth rate was $1.0 \AA \mathrm{s}^{-1}$. After $250 \mathrm{~nm}$-thick $\mathrm{SiO}_{2} \mathrm{NRs}$ deposition, $3 \mathrm{~nm}$-thick Pd film was sequentially deposited on the wafer at the original position $\left(0^{\circ}\right)$. These processes were repeatedly carried out until the $1 \mu \mathrm{m}$-thick v-SiO${ }_{2} \mathrm{NRs}$ were obtained. All the fabricated catalysts were annealed at $300^{\circ} \mathrm{C}$ for 30 minutes in a reducing atmosphere.

The morphology of the v-SiO 2 NRs@Pd nanostructure catalyst was characterized using a field-emission scanning electron microscopy (FESEM, Zeiss SUPRA 55VP). The transmission electron microscopy (TEM) and cross-sectional high-resolution TEM images were obtained using a $200 \mathrm{kV}$ filed-emission TEM (JEM-2100 F, JEOL). A focused ion beam (FIB, Helios 650) was utilized to prepare the samples for TEM analysis. The energy-dispersive X-ray spectroscopy (EDS) analysis was conducted to investigate the 
chemical mapping of the v-SiO ${ }_{2} \mathrm{NRs@Pd} \mathrm{nanostructured} \mathrm{catalyst.} \mathrm{Grazing-incidence} \mathrm{X-ray} \mathrm{powder}$ diffraction (GIXRD) was performed using PANalytical X'pert Pro X-ray diffractometer. The X-ray photoelectron spectroscopy (XPS) was carried out using AXIS Ultra DLD instrument (Kratos, U.K.) operating at a base pressure of $1.6 \times 10^{-10} \mathrm{mbar}$ at $300 \mathrm{~K}$. An inductively coupled plasma atomic emission spectrometer (ICP-AES, Varian 730ES) was used for the measurement of Pd amount. The products from the catalytic reactions were analyzed by a gas chromatography mass spectrometer (GCMS) using an Agilent Technologies 7693 Autosampler. The structures of the chemical products were identified using a nuclear magnetic resonance (NMR, 600MHz, High Resolution NMR Spectrometer, AVANCE Bruker).

\section{Results And Discussion}

The fabrication process for the v-SiO ${ }_{2} \mathrm{NRs@Pd} \mathrm{nanostructured} \mathrm{catalyst} \mathrm{using} \mathrm{an} \mathrm{electron} \mathrm{beam}$ evaporator is illustrated in Fig. 1. A multiple-step GLAD was utilized to deposit v-SiO ${ }_{2} \mathrm{NRs}$ on a $p$-type $\mathrm{Si}$ wafer and effectively adorning $\mathrm{Pd} \mathrm{NPs}$ on $\mathrm{v}-\mathrm{SiO}_{2} \mathrm{NRs}$, substantially improving the previously reported methods. ${ }^{39-41} \mathrm{SiO}_{2}$ was deposited on a $p$-type $\mathrm{Si}$ wafer tilted at $80^{\circ}$ and rotated with a speed of $80 \mathrm{rpm}$. After deposition of a $250 \mathrm{~nm}$-thick layer of $\mathrm{SiO}_{2} \mathrm{NRs}$, a $3 \mathrm{~nm}$-thick Pd layer was deposited at a tilt angle of $0^{\circ}$ on the v-SiO ${ }_{2} \mathrm{NRs}$. These steps were performed repeatedly until the total length of the $\mathrm{v}-\mathrm{SiO}_{2} \mathrm{NRs}$ reached to $1 \mu \mathrm{m}$. Finally, the fabricated catalysts were annealed at $300^{\circ} \mathrm{C}$ under a reducing atmosphere to furnish robustly stabilized Pd NPs.

As shown in Fig. 2a and b, v-SiO 2 NRs@Pd nanostructured catalyst was uniformly deposited on 4-inch Si wafer. The cross-sectional scanning electron microscopy image in Fig. $2 b$ shows highly one-dimensional structures of v-SiO $2 \mathrm{NRs@Pd} \mathrm{catalyst} \mathrm{on} \mathrm{the} \mathrm{Si}$ wafer substrate. The nanorods were separated from each other by elongated pores and their average diameter was about $60 \mathrm{~nm}$, improving the approachability of the Pd NPs and diffusion of the reactants and products. Due to their accessible nanostructure, reagents can efficiently permeate into and interact with the v-SiO ${ }_{2} \mathrm{NRs@Pd} \mathrm{nanostructured} \mathrm{catalyst.} \mathrm{To} \mathrm{clarify} \mathrm{the}$ existence of Pd NPs in the $\mathrm{v}_{-} \mathrm{SiO}_{2} \mathrm{NRs}$, GIXRD was performed which revealed the peaks assignable to the (111) and (200) reflections of the face-centered cubic (FCC) Pd NPs (Fig. 2c). XPS analysis was also carried out to confirm the oxidation state of Pd species in v-SiO 2 NRs@Pd nanostructured catalyst (Fig. 2d). The peaks corresponding to Si, O, and Pd are clearly observed in XPS spectra (Fig. S1). The Pd $3 d_{5 / 2}$ and $P d 3 d_{3 / 2}$ binding energies were found to be at 335.8 and $341.1 \mathrm{eV}$, respectively thus indicating the formation of zero-valent Pd species in v-SiO 2 NRs@Pd nanostructured catalyst.

To further analyze the nanostructured of $\mathrm{v}-\mathrm{SiO}_{2}$ NRs@Pd catalyst, TEM images were obtained as shown in Fig. 3a; four dense layers of Pd NPs were seen clearly deposited on $\mathrm{v}^{-\mathrm{SiO}_{2}} \mathrm{NRs}$. The Pd NPs were also observed in the side walls of the $\mathrm{v}-\mathrm{SiO}_{2} \mathrm{NRs}$. The energy dispersive spectroscopy (EDS) mapping on the v$\mathrm{SiO}_{2} \mathrm{NRs@Pd} \mathrm{nanostructured} \mathrm{catalyst} \mathrm{was} \mathrm{conducted.} \mathrm{Figure} \mathrm{3b-d} \mathrm{show} \mathrm{the} \mathrm{chemical} \mathrm{mapping} \mathrm{images}$ of $\mathrm{Si}, \mathrm{O}$, and $\mathrm{Pd}$ elements for the selected areas in Fig. 3a (orange dashed rectangle). For $\mathrm{Si}$ and $\mathrm{O}$ 
elements, a uniform distribution across the v- $\mathrm{SiO}_{2}$ NRs has been observed. However, Pd NPs were distributed in a layer-by-layer architecture with total of four layers. The Pd element observed in between the layers has been attributed to the Pd NPs on sidewalls of $\mathrm{v}^{-\mathrm{SiO}_{2}} \mathrm{NRs}$. Figure $3 \mathrm{e}-\mathrm{g}$ shows the high resolution TEM (HRTEM) images of Pd NPs on the surface (yellow rectangle) of the $\mathrm{SiO}_{2} \mathrm{NRs}$; Pd NPs were crystallized with the (111) preferred orientation. From the above results, it can be concluded that Pd NPs are efficiently decorated on the whole surface area of $\mathrm{SiO}_{2} \mathrm{NRs}$ and immobilized at desired locations on $\mathrm{SiO}_{2} \mathrm{NRs}$ through multiple-step GLAD method to realize a favorable spatial confinement. In other words, the catalytic active sites, $\mathrm{Pd} \mathrm{NPs}$, are well-refined on the $\mathrm{v}-\mathrm{SiO}_{2} \mathrm{NR}$ supports, resulting in more accessible catalytically activite sites on the v- $\mathrm{SiO}_{2} \mathrm{NRs@Pd} \mathrm{nanostructured} \mathrm{catalyst.}$

To further demonstrate the advantages of this physical architecture design, we fabricated $\mathrm{v}-\mathrm{SiO}_{2} \mathrm{NRs}$ decorated with $\mathrm{Au}$ and $\mathrm{Pd}$ alternately $\left(\mathrm{v}-\mathrm{SiO}_{2} \mathrm{NRs@Pd/Au}\right)$, using the same protocol. The four dense layers of metal NPs were observed on the v-SiO 2 NRs@Pd/Au nanostructured catalyst (Fig. S2). The EDS mapping on the v-SiO ${ }_{2} \mathrm{NRs@Pd/Au} \mathrm{nanostructured} \mathrm{catalyst} \mathrm{revealed} \mathrm{that} \mathrm{the} \mathrm{Pd} \mathrm{and} \mathrm{Au} \mathrm{NPs} \mathrm{were} \mathrm{layer-}$ by-layer deposited on $\mathrm{v}-\mathrm{SiO}_{2}$ NRs by turns. The HRTEM images also confirmed that the metal NPs were single crystalline in each layer for both, the Au and Pd NPs.

To confirm the oxidation state of Pd and Au NPs, XPS analysis was conducted. The peaks observed at 84.1, 87.8, 335.8, and $341.2 \mathrm{eV}$ corresponded to $\mathrm{Au} 4 f_{7 / 2}, \mathrm{Au} 4 f_{5 / 2}, \mathrm{Pd} 3 d_{5 / 2}$ and $\mathrm{Pd} 3 d_{3 / 2}$, respectively. This indicated that the Pd and Au NPs on the v-SiO 2 NRs@Pd/Au nanostructured catalyst were in zerovalent state. The similar catalyst was also synthesized on $\mathrm{SnO}_{2}$ supports using our strategy to broaden various type of supporting materials. As shown in Fig. S3, Pd NPs were well-decorated on v-SnO 2 NRs. These results illustrated that metal NPs could be stabilized on the designed sites on various supports using the multiple-step GLAD physical method.

Compared with previously reported fabrication processes for nanostructured catalysts, this approach has several advantages including favorable design, precise architecture engineering, and accessible metal nanocatalysts for the reactants, etc. ${ }^{42-44}$ Using wet chemical methods, mainly applied for nanostructured catalysts fabrication, catalytic sites are randomly distributed due to the lack of controllability and the need for complicated functionalization processes. ${ }^{45}$ In addition, it is very difficult to combine two or more metal NPs into the nanostructured support. However, this approach circumvents the limitations of previous reports for the catalysts on silicon wafers as the supporting materials via top-down fabrication process often is accompanied by harmful and toxic HF etching ${ }^{46}$ The present strategy provides the varying catalytically active sites precisely integrated on the support and their location can be controlled on nanoscale, thus improving the approachability of the catalytic NPs to reactants.

To verify the catalytic activity and chemoselectivity of the v-SiO ${ }_{2} \mathrm{NRs@Pd} \mathrm{nanostructured} \mathrm{catalyst,} \mathrm{we}$ conducted the hydrogenation of various substituted nitroaromatics at room temperature; a reaction that has important impact in many industries, including environmental safety. ${ }^{47-50}$ Our designed 
nanostructured catalyst presented superb catalytic activity for the selective hydrogenation of nitroaromatics in aqueous solution (Table 1 ). The procedure was accomplished under eco-friendly mild conditions, attaining high yields for the hydrogenation of substituted nitroaromatics to the corresponding aminoaromatics. The hydrogenation of nitroaromatics bearing two reducible groups could be achieved selectively to the amino moiety as the only product, while another reducible group was retained unchanged (Table 1, entries 4-6). In addition, achieving highly selective reduction of nitro groups of aromatic molecules possessing olefin bonds is very challenging as there is high probability for both the functional groups to be readily hydrogenated. Importantly, this process proceeded with high selectivity $(99 \%)$, reducing only nitro group (Table 1 , entry 8 ) as affirmed by nuclear magnetic resonance spectroscopy (Fig. S4).

To clarify the correlation between accessibility of Pd nanocatalysts and their activity, as a proof-ofconcept, control experiments were carried out using various nanostructured catalysts in the hydrogenation of nitrobenzene as a model reaction under identical reaction conditions (Table S1). Initially, only Pd NPs were deposited on silicon wafer by GLAD method aiming for simple recycling though dipping and removing of the silicon substrate possessing stable nanocatalysts. The reaction produced a low yield of (15\%) of aniline under optimized reaction conditions. FESEM images of this catalyst showed that the surface of the silicon wafer substrate was thoroughly covered by stacked Pd NPs without approachable opening for the diffusion of the reactants, resulting in low catalytic activity (Fig. S5). In addition, micro- and nano-space channels provide permeable paths to organic reagents, thus improving the accessibility of the Pd nanocatalysts. ${ }^{51-53}$ We also fabricated and evaluated the catalytic activity of $\mathrm{Pd} \mathrm{NPs}$ on $\mathrm{SiO}_{2}$ arrays having $200 \mathrm{~nm}$ height (Fig. S6). Interestingly, the $\mathrm{SiO}_{2}$ columns exposing Pd NPs could hydrogenate the nitro group of nitrobenzene in relatively higher yields (35\%). Although the prototype short length $\mathrm{SiO}_{2}$ arrays were partly aggregated, from this result we found that the Pd NPs stabilized on sturdy $\mathrm{SiO}_{2}$ support functioned to some extent effectively, which can be attributed to internal surface and pore diffusivity. ${ }^{54}$ Consequently, we designed and fabricated $\mathrm{SiO}_{2}$ arrays and aligning them separately from each other with $\sim 600 \mathrm{~nm}$ length possessing Pd NPs on their scaffolds as depicted in FESEM image (Fig. S7). Although the Pd NPs were located irregularly on the $\mathrm{SiO}_{2}$ arrays, their combination improved their interactions with the solvent and reactants via the accessible pores, promoting efficient catalytic reactions (Table S1, entry 3). Finally, we designed and developed fully arranged and assorted v-SiO 2 NRs@Pd catalyst having permeable tunnel nanostructure and elongated pores between $\mathrm{SiO}_{2}$ columns with four strata of attainable Pd NPs, and maximizing their catalytic activity in the given geometric configuration. Similarly, the alternatively four dense layers of Pd NPs and Au NPs in the v-SiO 2 NRs@Pd/Au nanostructured catalyst provided high catalytic activity in the model reduction. The combination and the activity of different nanocatalysts can easily be tuned and optimized by this method, controlling their locations on supports and distance between the nanocatalysts and the arrays. The controlled pores in the nanostructure provided facile adsorption of reactants to the Pd NPs and subsequent desorption of products from them. Additionally, a larger amount of Pd NPs can be decorated on the whole surface of $\mathrm{SiO}_{2} \mathrm{NRs}$ exposing large amount of accessible Pd sites for the catalytic 
reactions. Thus, v- $\mathrm{SiO}_{2} \mathrm{NRs@Pd} \mathrm{nanostructured} \mathrm{catalyst} \mathrm{could} \mathrm{expedite} \mathrm{Suzuki} \mathrm{coupling} \mathrm{reaction} \mathrm{of}$ various aryl halides with phenylboronic acid (Table 2); reaction of aryliodides and arylbromides with phenylboronic acid proceeded with excellent conversion in $3 \mathrm{~h}$ and $5 \mathrm{~h}$, respectively.

Recycling and reuse of the catalysts are crucial parameters because of the environmental impact and practical applications. Although many heterogeneous catalysts have been developed for the reduction of nitroaromatics, they generally entail very arduous recycling procedures such as centrifugation, filtration, laborious work-up purification, etc. However, v- $\mathrm{SiO}_{2} \mathrm{NRs@Pd} \mathrm{nanostructured} \mathrm{catalyst} \mathrm{could} \mathrm{be} \mathrm{easily}$ separated being withdrawn from the reaction mixture by tweezers, as they have a monolithic structure, a salient advantage of our catalyst. The durability and reusability of our catalyst was investigated for the hydrogenation of nitrobenzene. After completion of the reaction, the v-SiO ${ }_{2} \mathrm{NRs@Pd} \mathrm{nanostructured}$ catalyst was simply removed from the reaction mixture, rinsed with water, and reused in the next cycle. The activity of the catalyst remained stable even after seven consecutive reaction cycles, though there was a minuscule decline in activity of the catalyst (Table 3 ). As the catalytic activity of v-SiO ${ }_{2} \mathrm{NRs@Pd}$ nanostructured catalyst originates from its special architecture engineering of one body nanostructure, we precisely characterized the recovered v-SiO ${ }_{2} \mathrm{NRs@Pd} \mathrm{nanostructured} \mathrm{catalyst} \mathrm{by} \mathrm{EDX,} \mathrm{TEM,} \mathrm{and}$ HRTEM techniques (Fig. S8), affirming the constant stability of the designed catalyst after the recycling. Consequently, Pd leaching could be the reason for this catalytic decrease, which was supported by ICPAES analysis; negligible Pd species $\left(0.04 \mathrm{mg} \mathrm{L}^{-1}\right)$ was detected.

\section{Conclusions}

The novel synthesis of v-SiO 2 NRs@Pd nanostructured catalyst described in this study exhibited promising catalytic characteristics for the hydrogenation of nitroaromatics and the Suzuki crosscoupling reaction. The vertically aligned $\mathrm{SiO}_{2} \mathrm{NRs}$ provided an excellent platform for $\mathrm{Pd}$ nanocatalysts to be firmly cohered to create a monolithic structure, which is superior in the realization of a simple recycling process. Because this fabrication procedure is based on physical vapor deposition under vacuum conditions, it can be applied to various combinations of metal nanocatalysts and supporting nanostructures without complicated synthesis procedures often deployed in wet chemical methods. The effective design of nano-architectures for a monolithic nanocatalysts-attached $\mathrm{SiO}_{2} \mathrm{NRs}$ will expectedly open new perspectives toward the development of highly recyclable nanocatalysts for other catalytic reactions.

\section{Declarations}

\section{Acknowledgements}

This research was supported by National Research Foundation of Korea (NRF) funded by the Ministry of Science and ICT (2020M2D8A206983011). Furthermore, the financial supports of the Basic Science 
Research Program (2017R1A2B3009135) through the National Research Foundation of Korea is appreciated.

\section{Conflict of interest}

The authors declare no conflict of interest.

\section{References}

1. Hagen, J. Industrial catalysis: a practical approach (John Wiley \& Sons, 2015).

2. Ross, J. R. Heterogeneous catalysis: fundamentals and applications (Elsevier, 2011).

3. Polshettiwar, V. \& Varma, R. S. Green chemistry by nano-catalysis. Green Chem, 12, 743-754 (2010).

4. Polshettiwar, V., Baruwati, B. \& Varma, R. S. Self-Assembly of Metal Oxides into Three-Dimensional Nanostructures: Synthesis and Application in Catalysis. ACS Nano, 3, 728-736 (2009).

5. Ji, D. et al. Thin MoS2 nanosheets grafted MOFs-derived porous Co-N-C flakes grown on electrospun carbon nanofibers as self-supported bifunctional catalysts for overall water splitting. $J$. Mater. Chem. A, 5, 23898-23908 (2017).

6. Huang, W. et al. Highly Active Heterogeneous Palladium Nanoparticle Catalysts for Homogeneous Electrophilic Reactions in Solution and the Utilization of a Continuous Flow Reactor. J. Am. Chem. Soc, 132, 16771-16773 (2010).

7. Liu, L. \& Corma, A. Metal Catalysts for Heterogeneous Catalysis: From Single Atoms to Nanoclusters and Nanoparticles. Chem. Rev, 10, 4981-5079 (2018).

8. Cui, X., Li, W., Ryabchuk, P., Junge, K. \& Beller, M. Nanostructuring unlocks high performance of platinum single-atom catalysts for stable vinyl chloride production. Nat. Catal, 1, 376-385 (2020).

9. White, R. J., Luque, R., Budarin, V. L., Clark, J. H. \& Macquarrie, D. J. Supported metal nanoparticles on porous materials. Methods and applications. Chem. Soc. Rev, 38, 481-494 (2009).

10. Wang, Z. L., Li, C. \& Yamauchi, Y. Nanostructured nonprecious metal catalysts for electrochemical reduction of carbon dioxide. Nano Today, 11, 373-391 (2016).

11. Bavykina, A., Kolobob, N., Khan, I. S., Bau, J. A. \& Ramireza, Gascon, J. Metal-Organic Frameworks in Heterogeneous Catalysis: Recent Progress, New Trends, and Future Perspectives. Chem. Rev, 120, 8468-8535 (2020).

12. Goetjen, T. A. et al. Metal-organic framework (MOF) materials as polymerization catalysts: a review and recent advances. Chem. Commun, 56, 10409-10418 (2020).

13. Fan, Z. \& Zhang, H. Crystal phase-controlled synthesis, properties and applications of noble metal nanomaterials. Chem. Soc. Rev, 45, 63-82 (2016).

14. Hulsey, M. J., lim, C. W. \& Yan, N. Promoting heterogeneous catalysis beyond catalyst design. Chem. Sci, 11, 1456-1468 (2020). 
15. Mahmoud, M. A., Narayanan, R. \& El-Sayed, M. A. Enhancing Colloidal Metallic Nanocatalysis: Sharp Edges and Corners for Solid Nanoparticles and Cage Effect for Hollow Ones. Acc. Chem. Res, 46, 1795-1805 (2013).

16. Bakhtiarzadeh, Z. et al. Hydrothermal self - sacrificing growth of polymorphous MnO2 on magnetic porous-carbon (Fe304@Cg/MnO2): A sustainable nanostructured catalyst for activation of molecular oxygen. Mol. Catal, 509, 111603 (2021).

17. Gawande, M. B., Branco, P. S. \& Varma, R. S. Nano-magnetite (Fe304) as a support for recyclable catalysts in the development of sustainable methodologies. Chem. Soc. Rev, 42, 3371-3393 (2013).

18. Polshettiwar, V. et al. Magnetically Recoverable Nanocatalysts. Chem. Rev, 111, 3036-3075 (2011).

19. Zaera, F. Nanostructured materials for applications in heterogeneous catalysis. Chem. Soc. Rev, 42, 2746-2762 (2013).

20. Shokouhimehr, M., Shahedi Asl, M. \& Mazinani, B. Modulated large-pore mesoporous silica as an efficient base catalyst for the Henry reaction. Res. Chem. Intermed, 44, 1617-1626 (2018).

21. Astruc, Q. W. D. State of the Art and Prospects in Metal-Organic Framework (MOF)-Based and MOFDerived Nanocatalysis. Chem. Rev, 120, 1438-1511 (2020).

22. Perego, C. \& Millini, R. Porous materials in catalysis: challenges for mesoporous materials. Chem. Soc. Rev, 42, 3956-3976 (2013).

23. Ishida, T., Murayama, T., Taketoshi, A. \& Haruta, M. Importance of Size and Contact Structure of Gold Nanoparticles for the Genesis of Unique Catalytic Processes. Chem. Rev, 120, 464-525 (2020).

24. Hu, H. et al. Synthesis and stabilization of metal nanocatalysts for reduction reactions-a review. J. Mater. Chem. A, 3, 11157-11182 (2015).

25. Hong, K. et al. Palladium nanoparticles on assorted nanostructured supports: applications for Suzuki, Heck, and Sonogashira cross-coupling reactions. ACS Appl. Nano Mater, 3, 2070-2103 (2020).

26. Shi On the Synergetic Catalytic Effect in Heterogeneous Nanocomposite Catalysts. Chem. Rev, 113, 2139-2181 (2013).

27. Verma, P., Kuwahara, Y., Mori, K., Raja, R. \& Yamashita, H. Functionalized mesoporous SBA-15 silica: recent trends and catalytic applications. Nanoscale, 12, 11333-11363 (2020).

28. Sogut, E. G., Kuyuldar, H. A., karatas, Y., Gulcan, M. \& Sen, F. Single-walled carbon nanotube supported Pt-Ru bimetallic superb nanocatalyst for the hydrogen generation from the methanolysis of methylamine-borane at mild conditions. Sci. Rep, 9, 15724 (2019).

29. Zhang, K. et al. Pd modified prussian blue frameworks: Multiple electron transfer pathways for improving catalytic activity toward hydrogenation of nitroaromatics. Mol. Catal, 492, 110967 (2020).

30. Favier, I., Pla, D. \& Gómez, M. Palladium Nanoparticles in Polyols: Synthesis, Catalytic Couplings, and Hydrogenations. Chem. Rev, 120, 1146-1183 (2020).

31. Tao, R. et al. Porous organic polymer material supported palladium nanoparticles. J. Mater. Chem. A, 8, 17360-17391 (2020). 
32. Easson, M. W., Jordan, J. H., Bland, J. M., Hinchliffe, D. J. \& Condon, B. D. Application of Brown Cotton-Supported Palladium Nanoparticles in Suzuki-Miyaura Cross-Coupling Reactions. ACS Appl. Nano Mater, 3, 6304-6309 (2020).

33. Li, Z. et al. Well-Defined Materials for Heterogeneous Catalysis: From Nanoparticles to Isolated Single-Atom Sites. Chem. Rev, 120, 623-682 (2020).

34. Wang, C., Wang, Q., Fu, F. \& Astruc, D. Hydrogen Generation upon Nanocatalyzed Hydrolysis of Hydrogen-Rich Boron Derivatives: Recent Developments. ACC. Chem. Res, 53, 2483-2493 (2020).

35. Yang, N. et al. Adv. Mater, 30, 1803234 (2018).

36. Zhan, G., Li, P. \& Zeng, H. C. Architectural Designs and Synthetic Strategies of Advanced Nanocatalysts. Adv. Mater, 30, 1802094 (2018).

37. Shokouhimehr, M. et al. Magnetically retrievable nanocomposite adorned with Pd nanocatalysts: efficient reduction of nitroaromatics in aqueous media. Green Chem, 20, 3809-3817 (2018).

38. Shokouhimehr, M. Magnetically separable and sustainable nanostructured catalysts for heterogeneous reduction of nitroaromatics. Catalysts, 5, 534 (2015).

39. Jeon, J. M. et al. Vertically ordered SnO2 nanobamboos for substantially improved detection of volatile reducing gases. J. Mater. Chem. A, 3, 17939-17945 (2015).

40. Sanchez-Valencia, J. R., Longtin, R., Rossell, M. D. \& Gröning, P. Growth Assisted by Glancing Angle Deposition: A New Technique to Fabricate Highly Porous Anisotropic Thin Films. ACS Appl. Mater. Interfaces, 8, 8686-8693 (2016).

41. Barranco, A., Borras, A., Gonzalez-Elipe, A. R. \& Palmero, A. Perspectives on oblique angle deposition of thin films: From fundamentals to devices. Prog. Mater. Sci, 76, 59-153 (2016).

42. Yang, X. et al. Highly efficient reusable catalyst based on silicon nanowire arrays decorated with copper nanoparticles. J. Mater. Chem. A, 2, 9040-9047 (2014).

43. Katal, R. et al. Pd-Decorated CuO Thin Film for Photodegradation of Acetaminophen and Triclosan under Visible Light Irradiation. Adv. Mater. Interfaces, 5, 1801440 (2018).

44. Jin, Z., Liu, C., Qi, K. \& Cui, X. Photo-reduced Cu/CuO nanoclusters on TiO2 nanotube arrays as highly efficient and reusable catalyst. Sci. Rep, 7, 39695 (2017).

45. Goswami, A. et al. Pt nanoparticles decorated TiO2 nanotubes for the reduction of olefins. Appl. Mater. Today, 10, 86-92 (2018).

46. Yamada, Y. M. A., Yuyama, Y., Sato, T., Fujikawa, S. \& Uozumi, Y. A Palladium-Nanoparticle and Silicon-Nanowire-Array Hybrid: A Platform for Catalytic Heterogeneous Reactions. Angew. Chem. Int. $E d, 53,127-131$ (2014).

47. Shokouhimehr, M., Lee, J. E., Han, S. I. \& Hyeon, T. Magnetically recyclable hollow nanocomposite catalysts for heterogeneous reduction of nitroarenes and Suzuki reactions. Chem. Commun, 49, 4779-4781 (2013).

48. Nayebi, B. et al. Boron nitride-palladium nanostructured catalyst: efficient reduction of nitrobenzene derivatives in water. Nano express, 1, 030012 (2020). 
49. Shokouhimehr, M., Mahmoudi Gom-Yek, S., Nasrollahzadeh, M., Kim, A. \& Varma, R. S. Palladium nanocatalysts on hydroxyapatite: green oxidation of alcohols and reduction of nitroarenes in water. Appl. Sci, 9, 4183 (2019).

50. Kadam, H. K. \& Tilve, S. G. Advancement in methodologies for reduction of nitroarenes. RSC Adv, $\mathbf{5}$, 83391-83407 (2015).

51. Shokouhimehr, M. et al. Magnetically recyclable core-shell nanocatalysts for efficient heterogeneous oxidation of alcohols. J. Mater. Chem. A, 2, 7593-7599 (2014).

52. kalantari, E., Khalilzadeh, M. A., Zareyee, D. \& Shokouhimehr, M. Catalytic degradation of organic dyes using green synthesized Fe304-cellulose-copper nanocomposites. J. Mol. Struct, 1218, 128488 (2020).

53. Cheng, T., Zhang, D., Li, H. \& Liu, G. Magnetically recoverable nanoparticles as efficient catalysts for organic transformations in aqueous medium. Green Chem, 16, 3401-3427 (2014).

54. Iwamoto, M., Tanaka, Y., Sawamura, N. \& Namba, S. Remarkable Effect of Pore Size on the Catalytic Activity of Mesoporous Silica for the Acetalization of Cyclohexanone with Methanol. J. Am. Chem. Soc, 125, 13032-13033 (2003).

\section{Tables}

Table 1. Heterogeneous reduction of substituted nitroarenes catalyzed by v-SiO 2 NRs@Pd nanostructured catalyst. 


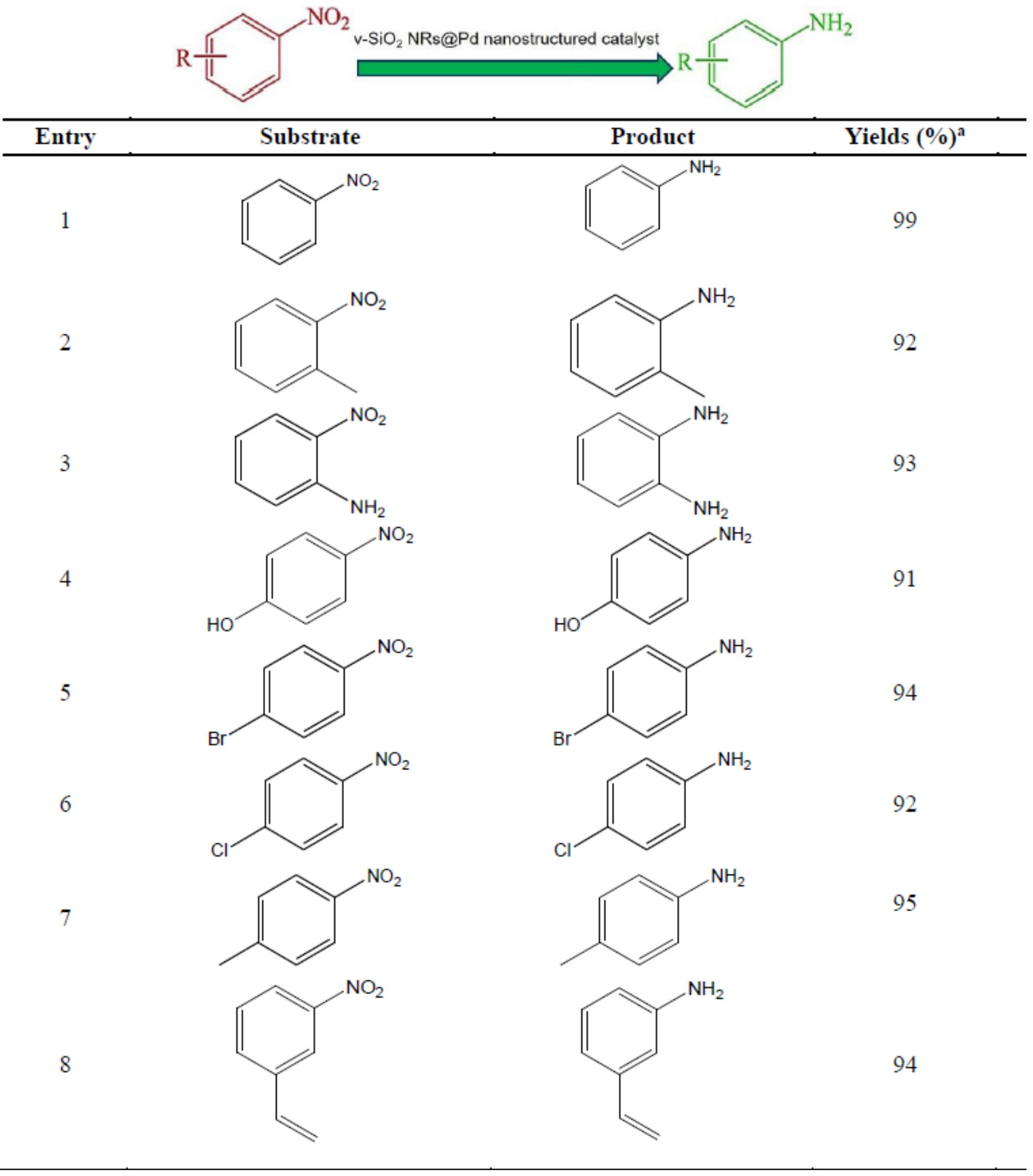

Reaction conditions: Substituted nitroarenes $(0.1 \mathrm{mmol}), \mathrm{NaBH}_{4}(0.12 \mathrm{mmol}), \mathrm{v}-\mathrm{SiO}_{2} \mathrm{NRs@Pd}$ nanostructured catalyst $(1 \mathrm{~mol} \% \mathrm{Pd}), \mathrm{H}_{2} \mathrm{O}(20 \mathrm{~mL})$, room temperature, and $1.5 \mathrm{~h}$. ${ }^{a}$ Yields were determined by GC-MS.

Table 2. Suzuki cross-coupling reactions catalyzed by v-SiO 2 NRs@Pd nanostructured catalyst. 


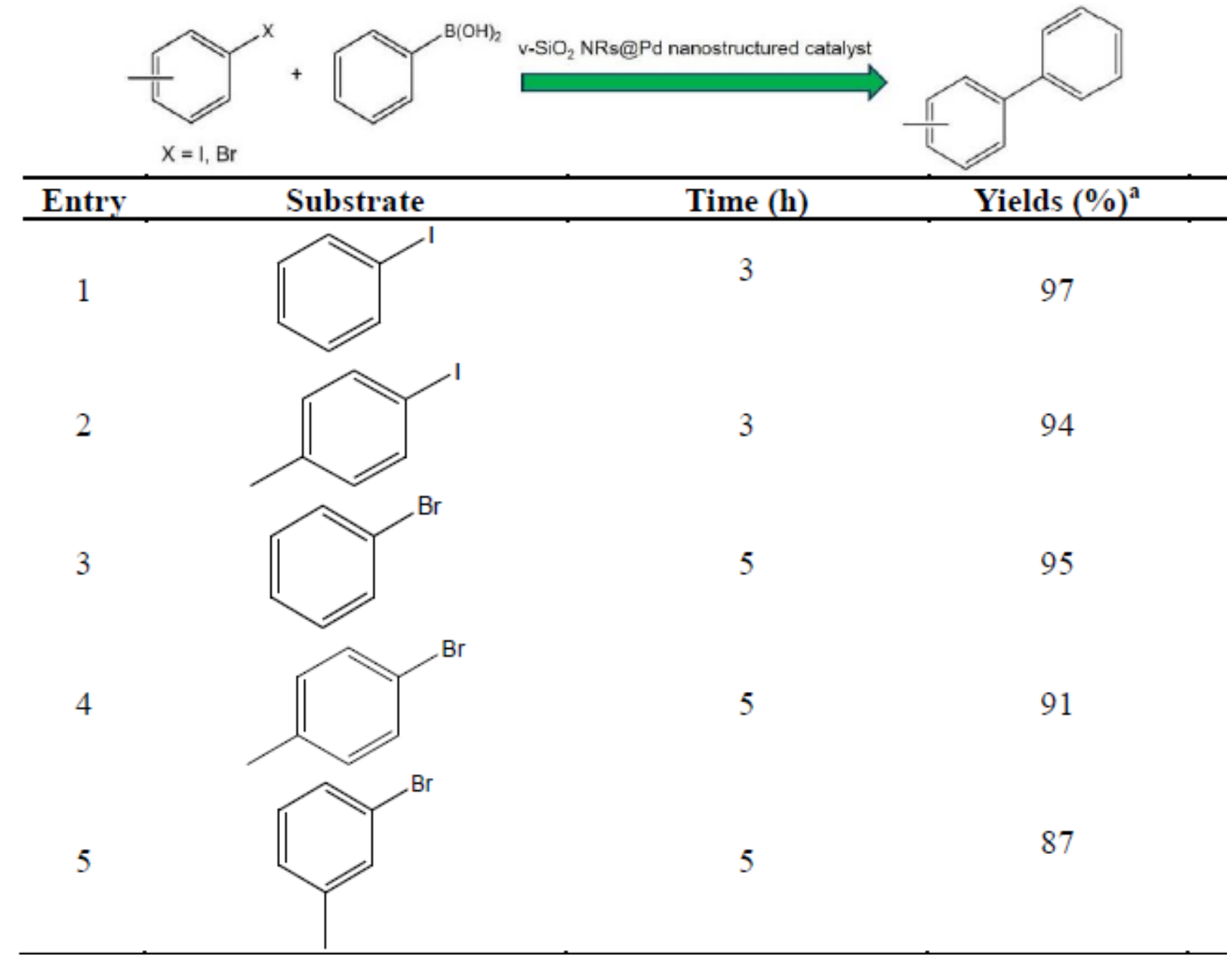

Reaction conditions: Aryl halide (0.1 mmol), phenylboronic acid (0.12 eq.),v-SiO 2 NRs@Pd nanostructured catalyst (1 mol\%), $\mathrm{K}_{2} \mathrm{CO}_{3}$ (1.5 eq.), DMF/ $\mathrm{H}_{2} \mathrm{O}(5: 1), 100{ }^{\circ} \mathrm{C}$. ${ }^{a}$ Yields were determined by GC-MS.

Table 3. Reuse of v-SiO 2 NRs@Pd nanostructured catalyst in the heterogeneous reduction of nitrobenzene. 


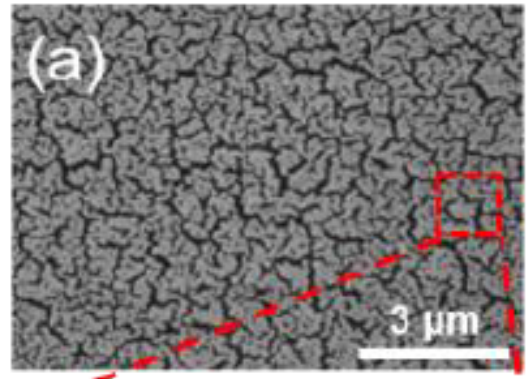

(c)
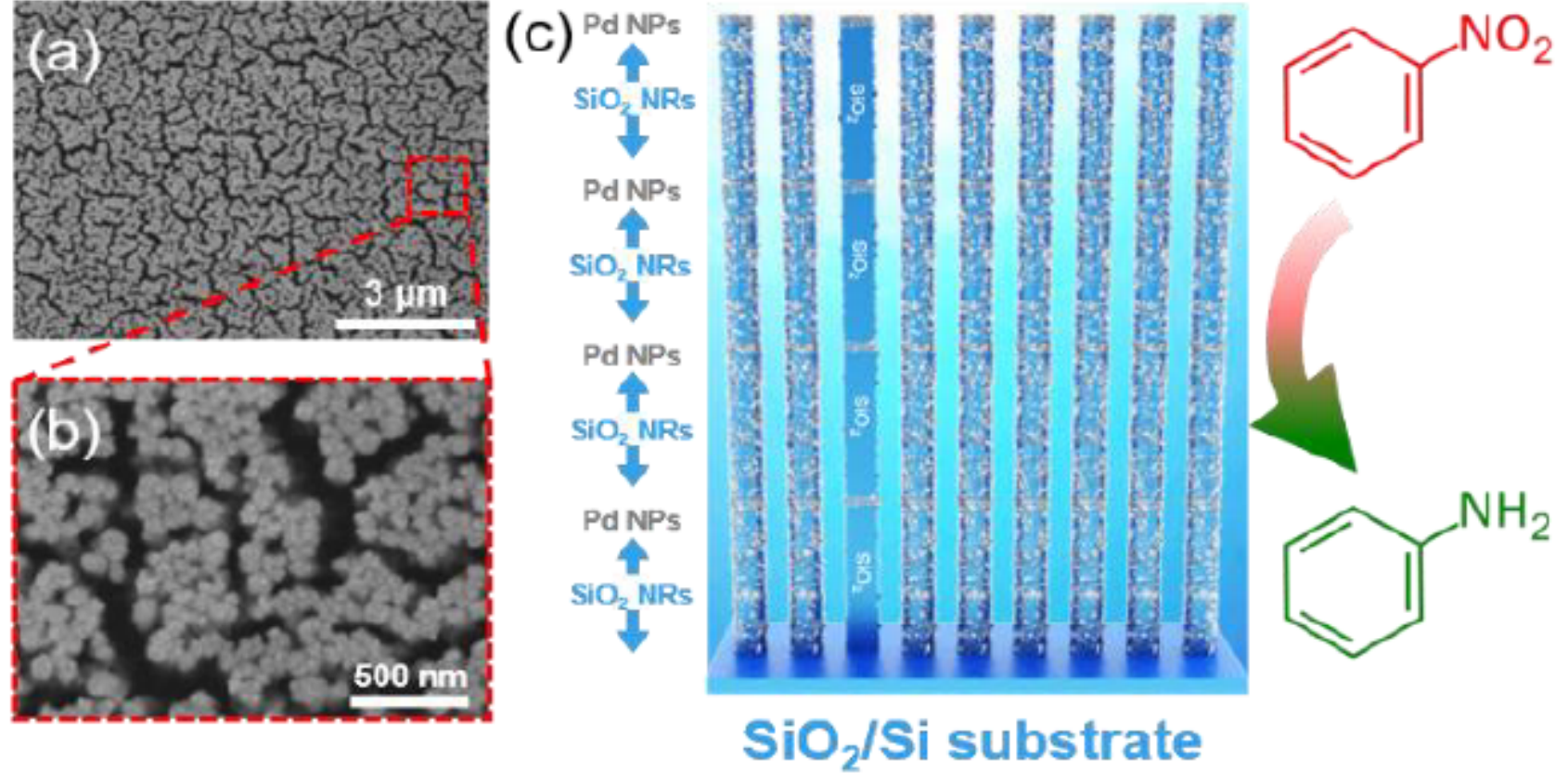

Cycle number 1 2 3

4
5 6

Yield of product (\%) ${ }^{\mathrm{a}} \quad 99$ 97 93 90 88 87 84

Reaction conditions: Substituted nitroarenes $(0.1 \mathrm{mmol}), \mathrm{NaBH}_{4}(0.12 \mathrm{mmol}), \mathrm{v}-\mathrm{SiO}_{2} \mathrm{NRs@Pd}$ nanostructure catalyst $(1 \mathrm{~mol} \% \mathrm{Pd}), \mathrm{H}_{2} \mathrm{O}(20 \mathrm{~mL})$, room temperature, and $1.5 \mathrm{~h}$. ${ }^{a}$ Yields were determined by GC-MS.

Figures 


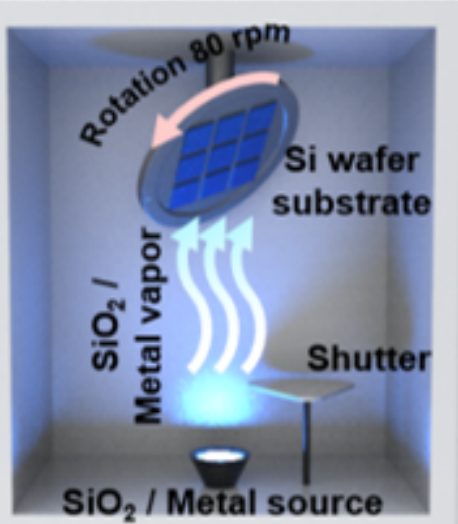

$\mathrm{SiO}_{2} /$ Metal source

Glancing angle deposition using e-beam evaporator
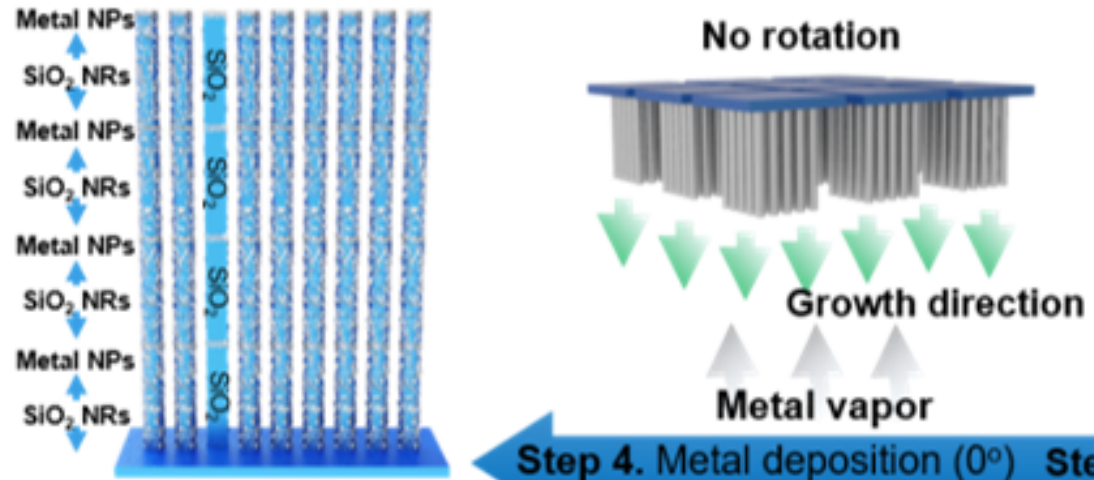

Step 4. Metal deposition $\left(0^{\circ}\right)$ Step 3. $\mathrm{SiO}_{2}$ NRs deposition $\left(80^{\circ}\right.$

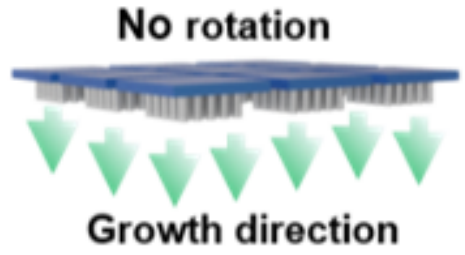

Metal vapor

Step 1. $\mathrm{SiO}_{2} \mathrm{NRs}$ deposition $\left(80^{\circ}\right)$ Step 2. Metal deposition $\left(0^{\circ}\right)$

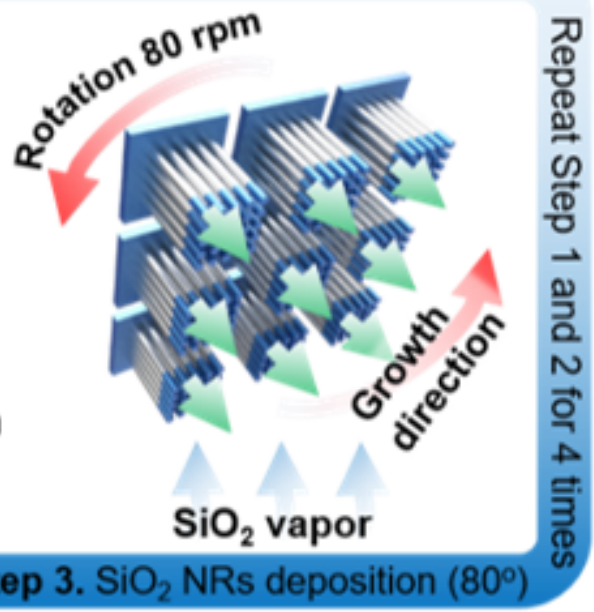

Annealing $\left(\mathrm{H}_{2} / \mathrm{N}_{2}, 300^{\circ} \mathrm{C}\right)$

Figure 1

Synthetic procedure of v-SiO2 NRs@Pd nanostructured catalyst. 

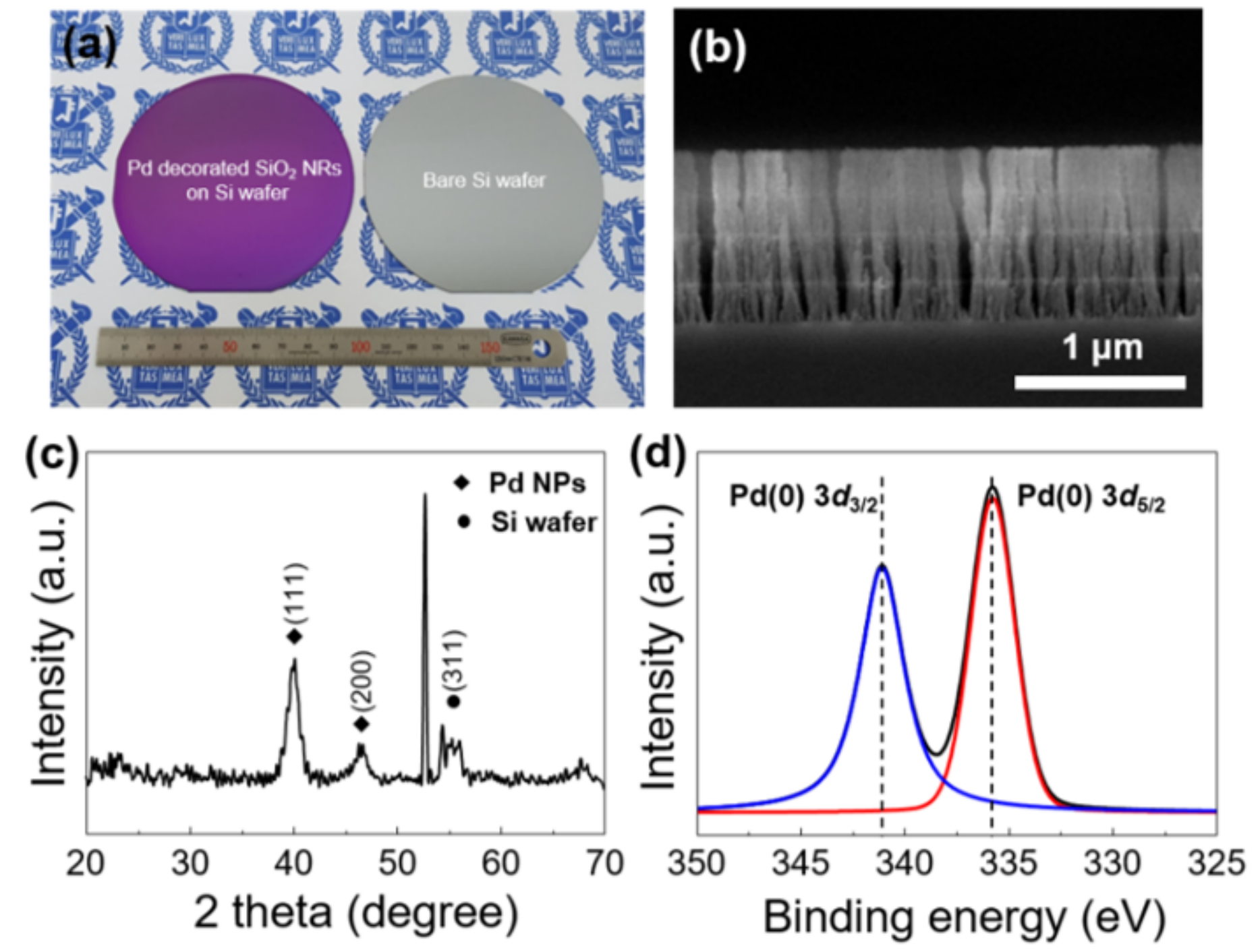

Figure 2

(a) A photographic image of v-SiO2 NRs@Pd nanostructured catalyst on p-type Si wafer (left) and bare ptype Si wafer. The diameter of the p-type Si wafers is 4 inches. (b) Cross-sectional SEM image of the vSiO2 NRs@Pd nanostructured catalyst. (c) GIXRD pattern of v-SiO2 NRs@Pd nanostructured catalysts on p-Si wafer. (d) XPS core level spectrum of Pd 3d for v-SiO2 NRs@Pd nanostructured catalyst. 


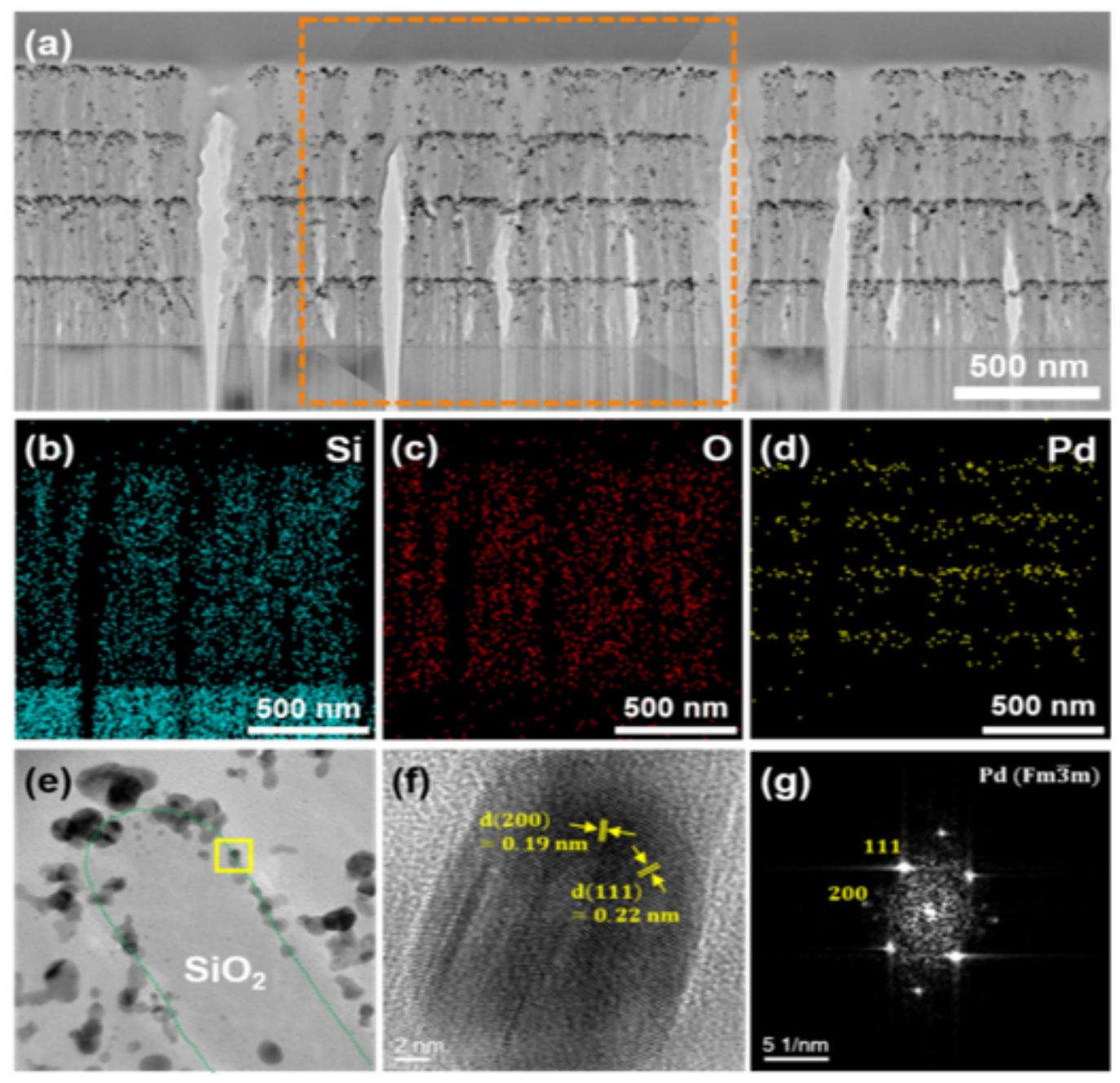

Figure 3

(a) Cross-sectional TEM image of the v-SiO2 NRs@Pd nanostructured catalyst. (b-g) EDS element maps of (b) Si, (c) 0, and (d) Pd for the v-SiO2 NRs@Pd nanostructured catalyst obtained from the orange dashed rectangle of (a). (e) HRTEM images of v-SiO2 NRs@Pd nanostructured catalyst. (f) HRTEM images and $(\mathrm{g})$ corresponding fast Fourier transform image of a Pd NP on SiO2 NRs obtained from the yellow rectangle in (e).

\section{Supplementary Files}

This is a list of supplementary files associated with this preprint. Click to download.

- 02Supportinginformation.docx 